throughout. These data were combined with the source location for the blood test, as well as the presence of coded comorbidity since the year 2000 .

Results There were 18171 patients left once patients discharged with a diagnosis of MI (122) and those suspected of MI (1707) by the clinical team were excluded. There were 4759 inpatients, 9280 outpatients and 4132 patients in the emergency department with $7.3 \%, 2.0 \%$ and $7.4 \%$ of these having an hs-cTn level above the manufacturer-provided ULN respectively. Table 1 demonstrates the prevalence of hs-cTn levels above the ULN in hospitalised patients by speciality. In terms of outpatients; cardiothoracic, renal and oncology/haematology patients were the most likely to have an hs-cTn above the ULN with $7.4 \%, 4.9 \%$ and $2.6 \%$ respectively above this level. There were 4265 (46\%) outpatients in whom there was at least one coded co-morbidity present. These patients were more likely to have an hs-cTn above the ULN than those without a coded co-morbidity $(2.8 \%$ versus $1.2 \%$, $\mathrm{P}<0.0001)$. Table 2 demonstrates the prevalence of outpatients with an hs-cTn above the ULN by coded co-morbidity.

Conclusion These data suggest that application of a manufacturer-derived 99th centile for hs-cTn assay to a hospital population may be flawed, particularly if any assumption is made that a result above this level may represent acute MI in patients without a classical history. Caution and a good understanding of the assay is imperative for accurate diagnosis and management of hospital patients with an apparently elevated level, but these results also raise interesting questions about what the levels actually indicate in patients in whom the suspicion $\mathrm{f}$ acute $\mathrm{Mi}$ is low. More data are required.

Conflict of Interest Educational support from BAYER

\section{HERITABILITY AND FAMILY-BASED GWAS ANALYSES OF THE CIRCULATING CERAMIDE, ENDOCANNABINOID, AND N-ACYL ETHANOLAMIDE LIPIDOME}

${ }^{1}$ Kathryn McGurk*, ${ }^{2}$ Bernard Keavney, ${ }^{1}$ Anna Nicolaou. ${ }^{1}$ The University of Manchester; ${ }^{2}$ Faculty of Biology, Medicine and Health, University of Manchester

\subsection{6/heartjnl-2019-BCS.101}

Introduction Lipids of the endocannabinoid (eCB), N-acyl ethanolamine (NAE), and ceramide (CER) classes are potential novel biomarkers of coronary artery disease and type-2 diabetes. Major-gene effects have been discovered for certain lipid species, notably lipoprotein(a). We sought to establish the heritability of eCB, NAE, and CER species, and identify DNA variants influencing their concentrations in plasma.

Methods We undertook heritability (QTDT, GCTA) and GWAS analyses (FaST-LMM) of $11 \mathrm{eCBs}$ and NAEs, and 37 CERs in 1,016 plasma samples from 196 British Caucasian families ascertained through a hypertensive proband, using targeted lipidomics by mass spectrometry, and Illumina 660WQuad genotyping.

Results Anandamide (AEA), a potent eCB, was found heritable (h2AEA $=32-35 \% ; \mathrm{P}<5.80 \times 10-11)$, however other less studied NAE species, presented higher estimates of heritability (h2NAEs $=41-79 \% ; \mathrm{P}<3.89 \times 10-13) .24-46 \%$ of the variation in potential biomarker CER is due to genetic factors $(\mathrm{P}<1.00 \times 10-7)$. GWAS identified associations with eQTLs of proteins involved in the metabolism of eCB and NAE (e.g. FAAH; PNAEDHEA $<6.33 \times 10-12$,) as well as CER
(SPTLC3; PCERN(24)S(18) <8.99 × 10-19) and novel loci implicated in cancer risk and non-alcoholic fatty liver disease (e.g. FBXO28; PCERN(24)S(19)ratio <1.95 $\times 10-8$, SULT1C4; PCERN(24)S(19) <8.99 × 10-19).

Two-sample Mendelian randomisation suggests that a variant in FAAH (rs324420) influencing the level of plasma NAEs (e.g. PNAEDHEA $<6.33 \times 10-12$ ) is causally associated with obesity, drug addiction, and anxiety. As an example, participants with the rs324420 AA genotype had a mean DHEA plasma concentration of $518 \mathrm{pg} / \mathrm{ml}$, which decreased by $27 \%$ in those with the AC genotype, and a further $11 \%$ in those carrying the CC genotype.

Conclusion We demonstrate for the first time estimates of heritability for this extended array of bioactive lipids, identify GWAS-significant SNPs associating with their levels in circulation, and implicate the lipid species studied here in cardiovascular disease, cancer, and drug addition. The results shown here can be used for prioritisation of lipid mediators for large-scale Mendelian Randomisation studies, and in the identification of causal metabolic pathways, novel diagnostics and drug targets for disease intervention.

Conflict of Interest None

\section{UNREPORTED CORONARY ARTERY CALCIFICATION ON UNGATED CT CHEST IMAGING: AN OPPORTUNITY MISSED?}

${ }^{1}$ David Hunter* ${ }^{*}$ Peter Van Rhijn, ${ }^{1}$ Richard Good, ${ }^{2}$ Colin Berry. ${ }^{1}$ NHS NWTC; ${ }^{2}$ NHS Greater Glasgow \& Clyde

\subsection{6/heartjnl-2019-BCS.102}

Introduction Coronary artery disease (CAD) remains the leading cause of morbidity and mortality worldwide1. Disabilityadjusted life years (DALY) are high in part due to the years of life lost and early onset of disease. Multiple therapeutic interventions including lipid modifying drugs, aggressive blood pressure control, detection and treatment of diabetes and additional lifestyle measures reduce the risk of cardiovascular events in patients with established vascular disease2. Coronary artery calcification (CAC) is initiated and propagated by endothelial dysfunction and vascular inflammation. Thus, the

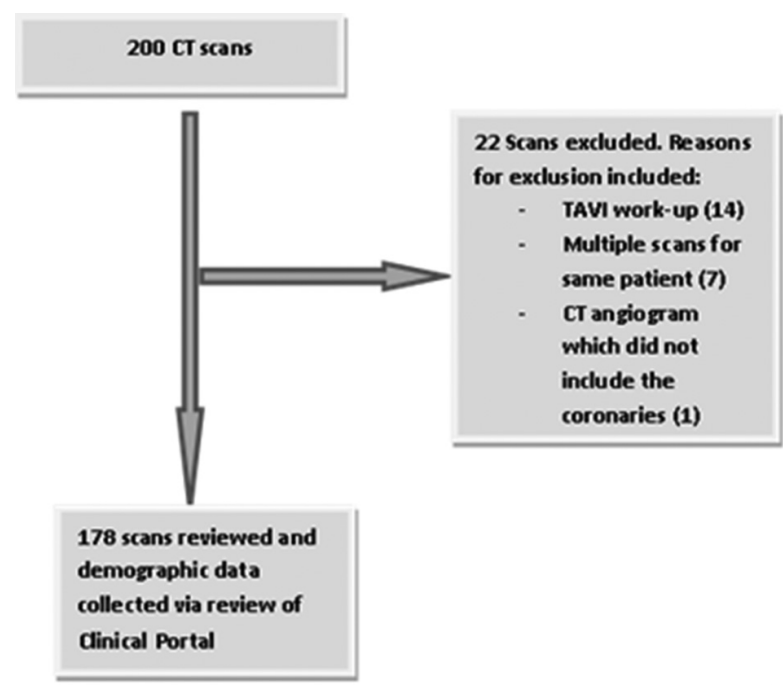

Abstract 105 Figure 1 